\title{
Reference-frequency generation by Raman-enhanced four-photon mixing
}

\author{
Sonia Martín López, Miguel González Herráez, Pedro Corredera, María Luisa Hernanz, \\ and Antonio Corróns
}

\begin{abstract}
Reference-frequency generation for optical fiber instrumentation is now restricted to the frequency bands in which the reference materials have well-resolved absorption lines. We study analytically and experimentally the possibility of generating reference wavelengths by use of Raman-enhanced four-photon mixing in an optical fiber. We show that it is possible to generate efficiently frequencies that are 10-40 $\mathrm{nm}$ away from the absorption bands of the usual reference materials: acetylene $\left({ }^{12} \mathrm{C}_{2} \mathrm{H}_{2}\right)$, hydrogen cyanide ( $\mathrm{HCN})$, and similar or derived species. As a demonstration we use an acetylene cell to generate reference frequencies that cover the whole $C(1530-1565 \mathrm{~nm})$ and some part of the $L(1565-1625 \mathrm{~nm})$ transmission bands of the optical fiber. (C) 2004 Optical Society of America

OCIS codes: $060.2300,060.4370,190.4380,190.5650$.
\end{abstract}

\section{Introduction}

In the modern communication systems that use dense wavelength-division multiplexing, the information is sent through the fiber in many frequency channels whose center frequency is separated from the others by 100,50 , or $25 \mathrm{GHz}$. The control of the center frequency of the channels must be very tight, so that there is no cross talk between channels. As the frequency difference between channels becomes smaller (compared with the values of the center frequency), the control of emission frequencies must be tighter.

The use of reference frequencies is interesting for the characterization of wavelength-measurement systems and of optical spectrum analyzers. These instruments are extremely necessary for the effective implementation of wavelength-division multiplexing systems. A fundamental method for the generation of reference wavelengths in the fiber bands of interest is based on the stabilization of lasers in the molecular or atomic absorption lines of some gases [basically

The authors are with the Instituto de Física Aplicada, Consejo Superior de Investigaciones Científicas, Serrano 144, 28006 Madrid, Spain. S. M. López's e-mail address is ltqm358@cetef.csic.es.

Received 27 September 2003; revised manuscript received 11 February 2004; accepted 2 March 2004.

0003-6935/04/153185-06\$15.00/0

(C) 2004 Optical Society of America acetylene $\left({ }^{12} \mathrm{C}_{2} \mathrm{H}_{2}\right)$, hydrogen cyanide $(\mathrm{HCN})$, and similar or derived species $\left.{ }^{1-4}\right)$.

The main problem with this method is that we are limited by the use of reference gas cells at frequencies in which they have well-resolved absorption lines. The International Telecommunication Union (ITU) has established a group of useful transmission bands of the fiber. If we want to obtain reference lines that cover all the ITU's bands it is necessary to use more than one cell or, more commonly, to use a reference etalon to extend the wavelength calibration. The first approach is expensive because it requires several reference cells with accurate traceability. The etalon approach has greater error at frequencies far from the reference frequency. Here we present a theoretical and experimental study of the possibility of generating reference wavelengths by Ramanenhanced four-wave mixing (FWM).5, The importance of this paper lies in its showing an efficient way to generate reference frequencies in bands that lie far from the absorption bands of the common reference materials, with an uncertainty that is basically comparable to that of the stabilization. At the basis of our method is FWM (or four-photon mixing) in optical fibers. Through this process two frequencies, $\omega_{1}$ and $\omega_{2}$, injected into the fiber generate another wave at frequency $\omega_{3}=2 \omega_{2}-\omega_{1}$. If we can stabilize $\omega_{1}$ and $\omega_{2}$ in absorption lines of our reference material we can generate through this process a third wave with a precisely known frequency. If the configuration of the experiment is adequately chosen, we can possibly generate this wave in frequency regions that lie rel- 
atively far from the absorption bands of the selected reference material. We demonstrate also that Raman assistance strongly improves the results of the generation. For example, we use only one ${ }^{12} \mathrm{C}_{2} \mathrm{H}_{2}$ cell to generate reference frequencies along the whole $C$ band and some part of the $L$ band of the fiber (up to $1574 \mathrm{~nm}$ ). With the use of Raman pump lasers with more power or of an absorption cell closer to the $C$ band center (for instance, ${ }^{13} \mathrm{C}_{2} \mathrm{H}_{2}$ ) it would be possible to generate reference wavelengths over the whole $C$ and $L$ bands, with the need for only a single reference material.

This paper is organized as follows: Section 2 is devoted to explaining the theoretical analysis of the process of Raman-enhanced FWM; in Section 3 we describe the experimental conditions and results; Section 4 is devoted to describing the potential application of FWM to the generation of reference wavelengths close to ITU frequencies along the $C$ band. Finally, in Section 5 we summarize the basic conclusions obtained from this study.

\section{Theoretical Analysis}

The response of an optical fiber to light becomes nonlinear for intense electromagnetic fields. ${ }^{7} \quad$ There are two main factors that contribute to this response: electronic response and molecular response. The electronic component is almost instantaneous (a few electronic cycles), and its effects on the light are elastic (i.e., no energy is exchanged between the electromagnetic field and the dielectric medium). This component of the nonlinear response is responsible for FWM, a phenomenon in which two input photons are annihilated to generate two other photons of different frequencies following energy-conservation rules. The molecular response of the material (silica) is much slower than the electronic component of the response (of the order of tens of femtoseconds), and its effects are inelastic (i.e., some energy is transferred from the electromagnetic wave to the dielectric medium). This component is responsible for the Raman effect in which one pump photon is annihilated to produce a photon of lower energy, following photon number conservation rules (the rest of the energy is transferred to an optical phonon).

We can analyze these two effects together by using third-order electric susceptibility $\left[\chi^{(3)}\right]$. Although in general doing so would involve a difficult tensor treatment, we can make it scalar by using the common assumption of linear, aligned polarizations along the fiber distance. This substitution is not strictly valid in conventional non-polarization-maintaining fibers, but the usual results when this assumption is made are normally in good agreement with the experiments. Experimentally we get polarization alignment at the input end of the fiber by introducing polarization controllers.

For polarization alignment we can preserve only the $\chi_{x x x x}^{(3)}$ component of the susceptibility tensor:

$$
\chi^{(3)}(\Omega)=\chi_{e}^{(3)}+\chi_{R}^{(3)}(\Omega),
$$

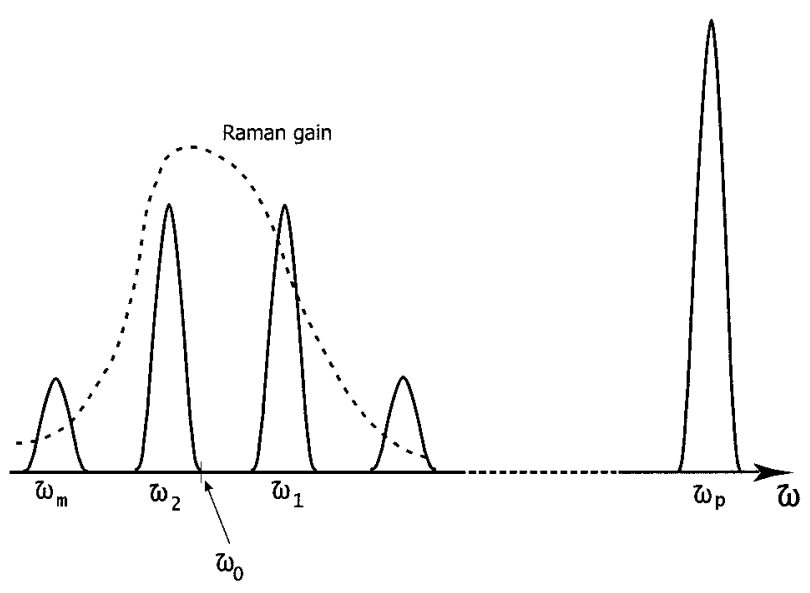

Fig. 1. Wavelength arrangement.

where the electronic $\left[\chi_{e}^{(3)}\right]$ and the Raman $\left[\chi_{R}^{(3)}\right]$ contributions appear. $\Omega$ is the separation between pump and probe frequencies. As in the research reported in Ref. 8, we approximate the Raman contribution to the nonlinear susceptibility by using a Lorentzian function:

$$
\chi_{R}(\Omega)=\frac{\rho}{1-\rho} \chi_{e} \frac{\tau_{1}^{2}+\tau_{2}^{2}}{\tau_{1}^{2}+\tau_{2}^{2}\left(1+i \tau_{1} \Omega\right)},
$$

where $\rho$ is the fractional Raman contribution to the nonlinear coefficient (typically 0.18 ). The adjustable parameters $\tau_{1}$ and $\tau_{2}$ take the values 32 and 12 $\mathrm{fs}$, respectively.

For our specific problem we consider two continuous waves of wavelengths $\lambda_{1}$ and $\lambda_{2}$ propagating along the fiber. We denote their slowly varying amplitudes $A_{1}$ and $A_{2}$, respectively. Simultaneously, we consider a continuous, counterpropagating Raman pump with a slowly varying amplitude $A_{p}$ at a wavelength $\lambda_{p}$. Figure 1 shows the locations of all the waves that are involved in our problem. The three input wavelengths verify the relation that follows: $\lambda_{p}<\lambda_{1}<\lambda_{2}$. We consider $\lambda_{2} \sim \lambda_{0}$, where $\lambda_{0}$ is the zero-dispersion wavelength of the fiber. Through FWM we get a new frequency component, $\omega_{m}$, verifying that $\omega_{m}=2 \omega_{2}-\omega_{1}$. The frequencyconversion efficiency depends on the optical phase difference among the four photons involved in the process $\left(\Delta \beta=2 \beta_{2}-\beta_{1}-\beta_{m}\right)$. FWM is efficient only when this phase mismatch is close to zero $(\Delta \beta \sim 0)$. We can say that this occurs basically when pump wavelength $\lambda_{2}$ is close to $\lambda_{0}$ and frequency difference $\omega_{2}-\omega_{1}$ is reasonably small. We consider that $\lambda_{1}, \lambda_{2}$, and $\lambda_{m}$ experience the Raman gain generated by the pump at $\lambda_{p}$. We also consider that the initial powers $P_{1}$ and $P_{2}$ are much lower than the Raman threshold, so all Raman processes induced by them can be considered negligible. All these assumptions are basically valid under our experimental conditions and help to simplify the solution greatly. $A_{m}$ is the slowly varying amplitude of the wavelength gener- 
ated through FWM. We assume that this amplitude is much smaller than the FWM pumps; thus

$$
\left|A_{p}\right| \gg\left|A_{1}\right|, \quad\left|A_{2}\right| \gg\left|A_{m}\right| .
$$

Implicitly, we assume that the depletion of the Raman pump and the FWM pumps is negligible. With all these considerations taken into account, it is possible to derive the propagation equations for all the waves:

$$
\begin{aligned}
\frac{\mathrm{d} A_{p}}{\mathrm{~d} z}= & \frac{\alpha}{2} A_{p}-i \gamma(0)\left|A_{p}\right|^{2} A_{p}, \\
\frac{\mathrm{d} A_{1}}{\mathrm{~d} z}= & -\frac{\alpha}{2} A_{1}-i 2 \gamma\left(\Omega_{1}\right)\left|A_{p}\right|^{2} A_{1}, \\
\frac{\mathrm{d} A_{2}}{\mathrm{~d} z}= & -\frac{\alpha}{2} A_{2}-i 2 \gamma\left(\Omega_{2}\right)\left|A_{p}\right|^{2} A_{2}, \\
\frac{\mathrm{d} A_{m}}{\mathrm{~d} z}= & -\frac{\alpha}{2} A_{m}-i 2 \gamma\left(\Omega_{m}\right)\left|A_{p}\right|^{2} A_{m} \\
& +i \gamma_{e} A_{2}^{2} A_{1} * \exp (i \Delta \beta z),
\end{aligned}
$$

where

$$
\begin{aligned}
\gamma\left(\Omega_{i}\right) & =\frac{3 \omega_{p}}{8 n c A_{\mathrm{eff}}}\left[\chi_{e}+\chi_{R}\left(\Omega_{i}\right)\right], \\
\gamma_{e} & =\frac{3 \omega_{2}}{8 n c A_{\mathrm{eff}}} \chi_{e} .
\end{aligned}
$$

In Eqs. (8) and (9), $n$ and $A_{\text {eff }}$ are the refractive index and the effective area of the fiber at the corresponding frequency, respectively. As initial conditions we take

$$
\begin{aligned}
\left|A_{p}(L)\right|^{2} & =P_{p}(L), \\
\left|A_{1}(0)\right|^{2} & =P_{1}(0), \\
\left|A_{2}(0)\right|^{2} & =P_{2}(0), \\
A_{m}(0) & =0 .
\end{aligned}
$$

At this point we take $\alpha=0$ (i.e., a fiber with no losses). The immediate solutions then are

$$
\begin{aligned}
A_{p}= & \sqrt{P_{p}(L)} \exp \left[-i z \gamma(0) P_{p}(L)\right], \\
A_{1}= & \sqrt{P_{1}(0)} \exp \left[2 i z \gamma\left(\Omega_{1}\right) P_{p}(L)\right], \\
A_{2}= & \sqrt{P_{2}(0)} \exp \left[2 i z \gamma\left(\Omega_{2}\right) P_{p}(L)\right], \\
A_{m}= & a\left\{\exp \left[4 i z \gamma\left(\Omega_{m}\right) P_{p}(L)\right]\right. \\
& \times \exp \left[-2 i z \gamma^{*}\left(\Omega_{1}\right) P_{p}(L)\right] \\
& \left.\times \exp (i \Delta \beta z)-\exp \left[2 i z \gamma\left(\Omega_{m}\right) P_{p}(L)\right]\right\},
\end{aligned}
$$

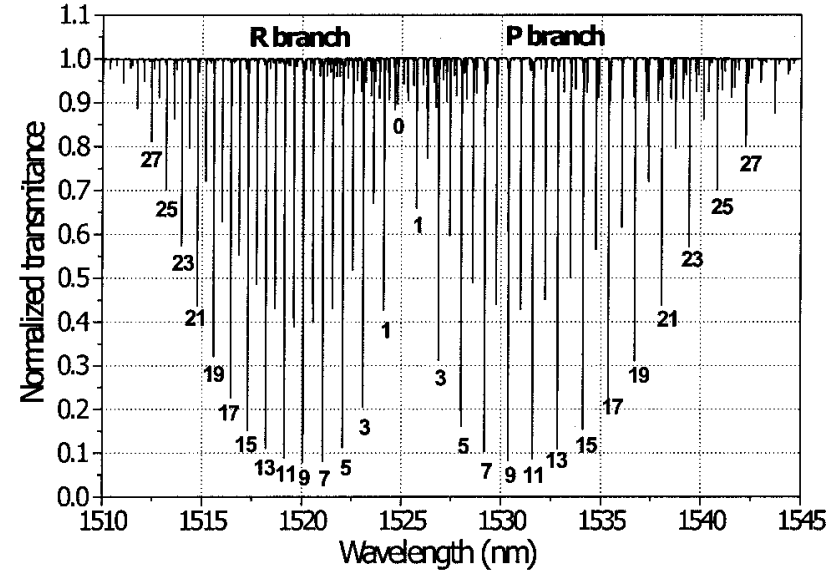

Fig. 2. Absorption spectrum of the ${ }^{12} \mathrm{CH}_{2}$ cell contained in reference material $2517 \mathrm{a}$.

where

$$
\begin{aligned}
& a=\frac{i \gamma_{e} P_{2}(0) \sqrt{P_{1}(0)}}{b+c+d+e}, \\
& b=-2 i \gamma\left(\Omega_{m}\right) P_{p}(L), \\
& c=4 i \gamma\left(\Omega_{2}\right) P_{p}(L), \\
& d=-2 i \gamma^{*}\left(\Omega_{1}\right) P_{p}(L), \\
& e=i \Delta \beta .
\end{aligned}
$$

To get numerical results from Eqs. (4)-(7) we must give some values to the fiber parameters. We assume that we have $5 \mathrm{~km}$ of fiber with a nonlinear coefficient of $\gamma_{e}=2.5 \mathrm{~W}^{-1} \mathrm{~km}^{-1}$. We estimate the Raman contribution, taking $\rho=0.13$. This value was obtained by measurement of the Raman gain factor in the fiber and comparison of the result with the expected $\gamma_{e}$. We have assumed that $n$ and $A_{\text {eff }}$ keep constant over the wavelength range of interest. The pump wavelength is fixed at $\lambda_{p}=1455 \mathrm{~nm}$, and its power is $P_{p}=400 \mathrm{~mW}$. For the FWM pumps we consider input powers $P_{1}=2 \mathrm{~mW}$ and $P_{2}=10 \mathrm{~mW}$; their wavelengths are $\lambda_{2}=1542.25 \mathrm{~nm}$ (which basically corresponds to the P27 line of the absorption spectrum of ${ }^{12} \mathrm{C}_{2} \mathrm{H}_{2}$; see Fig. 2) and $\lambda_{1}$, which varies from 1500 to $1540 \mathrm{~nm}$ (covering all the band in which the acetylene has well-resolved absorption lines), at $0.05 \mathrm{~nm}$ wide intervals. For calculus of the phase mismatch by unit length $\Delta \beta$ we make a fourth-order approximation:

$$
\Delta \beta=\beta_{2}\left(\omega_{1}-\omega_{2}\right)^{2}+\frac{\beta_{4}}{12}\left(\omega_{1}-\omega_{2}\right)^{4} .
$$

To evaluate this approximation we need the second- and fourth-order derivatives of $\beta$ at $\omega_{2}$, i.e., $\beta_{2}$ and $\beta_{4}$. Their values are fixed at $-2.5 \times 10^{-1}$ $\mathrm{ps}^{2} / \mathrm{km}$ and $2.5 \times 10^{-4} \mathrm{ps}^{4} / \mathrm{km}$, respectively. The value of $\beta_{2}$ corresponds to the measured dispersion in our experimental conditions, whereas the value of $\beta_{4}$ 


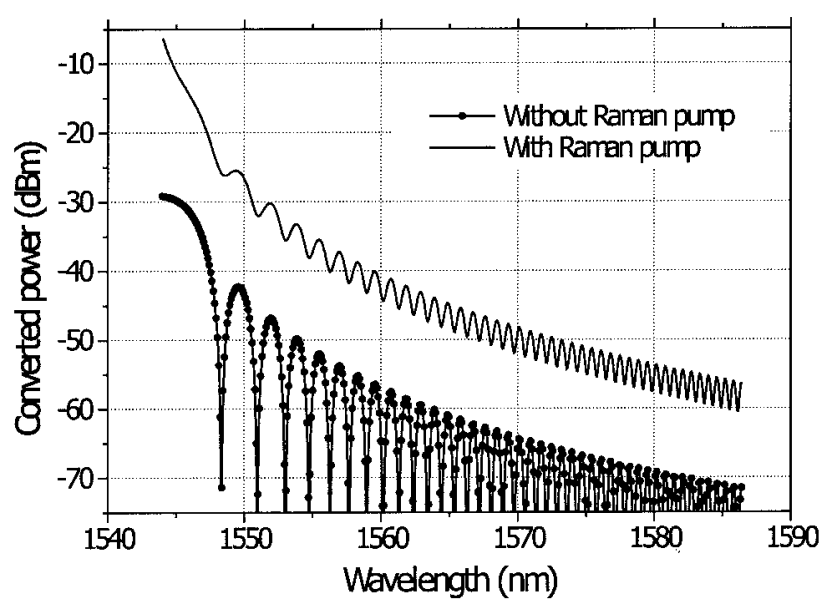

Fig. 3. Theoretical estimation of FWM power related to wavelength, with and without a Raman pump.

was extracted from typical values that appear in the literature. The contribution of $\beta_{4}$ to $\Delta \beta$ is rather small, although it is noticeable in the wavelengths that lie far away from the pump.

Figure 3 shows the relation between FWM wavelength $\lambda_{m}$ and its power, $P_{m}$. The two curves compare the FWM spectrum in the presence and in the absence of a Raman pump. There is an overall gain in the converted power given by the Raman pump all along the band of interest. As the Raman pump power grows, the difference between the two curves increases. In our conditions, the mean gain in the converted power that is due to the presence of the Raman pump is approximately $15-20 \mathrm{~dB}$. We can also observe that in absence of Raman pump power there are some points at which $P_{m}$ is zero, corresponding to the points that verify that $\Delta \beta L=n 2 \pi$. When the pump power is nonzero, $P_{m}$ gets a much more nearly uniform aspect, and the lobes appear drastically flattened. The aspect of the lobes gets flatter as the Raman pump power grows. So at this point we can conclude that, when the Raman pump is present, the output phase-matching conditions are less important in FWM. As we increase the pump power, that importance decreases.

\section{Experimental Results}

To demonstrate the theoretical results we built the experiment that appears in Fig. 4. The experimental conditions fit, almost exactly, the theoretical situation described in Section 2. The values of the input powers are those used in the previous calculations $\left(P_{1}=2 \mathrm{~mW}, P_{2}=10 \mathrm{~mW}\right.$, and $P_{p}=400 \mathrm{~mW}$; see above). The characteristics of the fiber used in the experiment are the same as those used in the previous calculations. The outputs of the two tunable lasers are delivered either to the fiber or to the stabilization loop by switches $\mathrm{SW}_{1}$ and $\mathrm{SW}_{2}$. Through a control program we can stabilize the two lasers in different absorption lines of the gas cell. In this way it is possible to update the state of the lasers in periods not longer than a few hundreds of milliseconds. The laser linewidths are approximately $150 \mathrm{kHz}$, to ensure that the stabilization is precise enough. The FWM generated at $\omega_{m}=2 \omega_{2}-\omega_{1}$ will have a well-known frequency according to our reasoning described above. We can estimate the uncertainty of the newly generated frequency by knowing the uncertainty in the stabilization of the two lasers to their corresponding absorption lines (see Section 4 below for details).

To compare with theoretical results, we lock the wavelength of laser TLS2 on the P27 absorption line of ${ }^{12} \mathrm{C}_{2} \mathrm{H}_{2}$, which is centered at $\lambda_{2}=1542.2508 \mathrm{~nm}$. With laser TLS1 we sweep the entire band from 1500 to $1540 \mathrm{~nm}$ while we measure the converted power. As a reference cell we use Standard Reference Material 2517a, ${ }^{9}$ whose spectrum is plotted in Fig. 2. ${ }^{12} \mathrm{C}_{2} \mathrm{H}_{2}$ has absorption lines at $1511-1540 \mathrm{~nm}$, so we are capable of generating lines with precisely known frequencies that cover the whole $C$ band and some part of the $L$ band (up to $1574 \mathrm{~nm}$ ). From Fig. 5 we can see the experimental measure of the FWM power against wavelength, with and without a Raman pump. The resemblance between the theoretical and the experimental results is good, when one takes

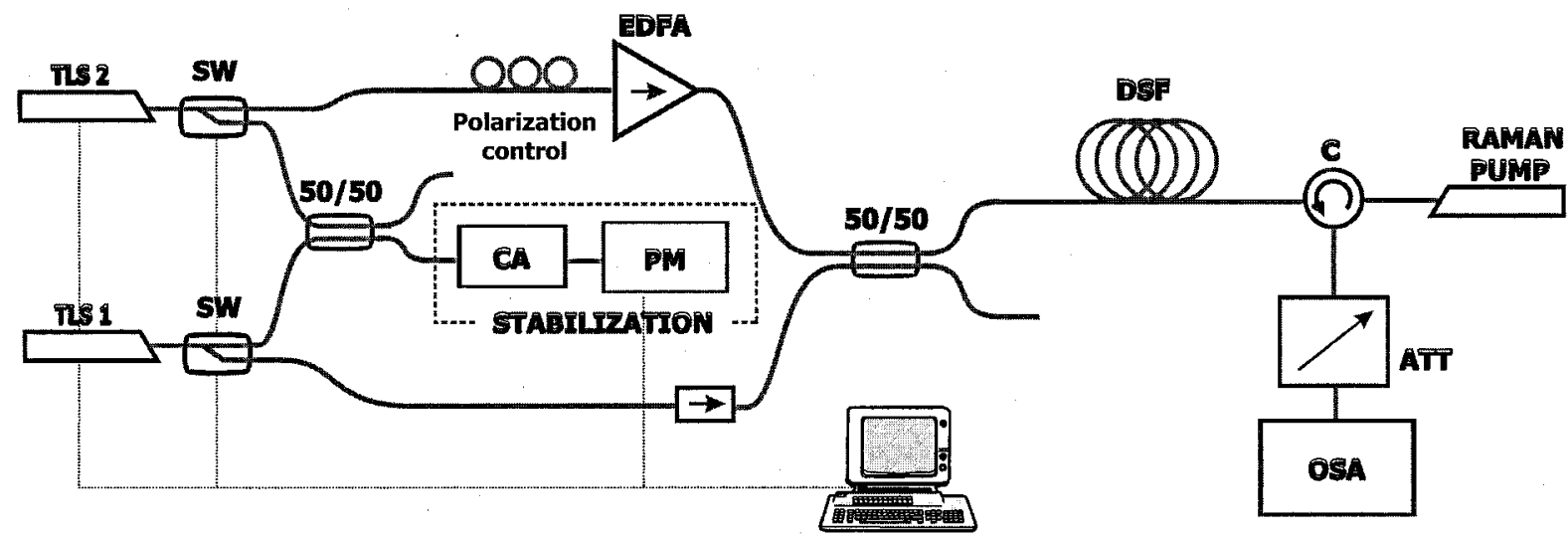

Fig. 4. Schematic of the experiment: TLSs, tunable lasers; CA, ${ }^{12} \mathrm{C}_{2} \mathrm{H}_{2}$ cell; EDFA, erbium-doped fiber amplifier; PM, powermeter; ATT, attenuator; OSA, optical spectrum analyzer; DSF, dispersion-shifted fiber $\left(\lambda_{0}=1540.9 \mathrm{~nm}\right)$; C, circulator; SWs, switches. 


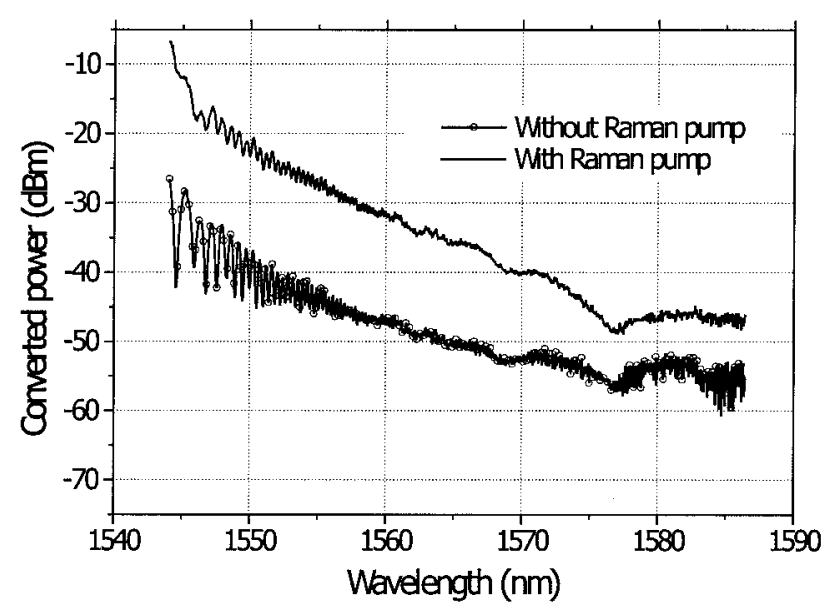

Fig. 5. Experimental results.

into account the many approximations that have been made in the theoretical treatment. Notice the 20-dB gain between zero Raman pump power and full Raman pump power. We can see that, along the entire band in which we are interested (1544-1574 $\mathrm{nm}$ ), generation with a Raman pump produces powers higher than $-50 \mathrm{dBm}$. Also note that, in some points close to $1544 \mathrm{~nm}$, the generated power is as high as $-5 \mathrm{dBm}$.

\section{Application to International Telecommunication Union Frequencies}

The results just described demonstrate that, if we use the ${ }^{12} \mathrm{C}_{2} \mathrm{H}_{2}$ absorption cell, we can generate reference frequencies in the region $1540-1574 \mathrm{~nm}$. In this section we give a few examples of frequency settings that can be chosen to produce reference frequencies in this region that are close to ITU nominal frequencies. In Table 1 we list some input frequencies (with the corresponding absorption lines), in each case together with the generated frequency and the uncertainty obtained for the new frequency, $\Delta f_{\mathrm{FWM}}$. The uncertainty of the new frequency depends only on the uncertainties in the knowledge of the initial frequencies $\left(\Delta f_{1}\right.$ and $\left.\Delta f_{2}\right)$. The uncertainties of the original frequencies depend basically on the linewidth of each absorption and on the absorption of each line, and thus they vary from line to line. The contribution of the laser's linewidth to the uncertainty is basically negligible in our conditions. Note that the uncertainty of the frequencies generated is always below $500 \mathrm{MHz}$.

A problem might arise in our setup when we try to make this configuration more efficient. FWM causes the linewidth of the generated wave to be several times bigger than those of the original waves. In the present configuration of the experiment, no problem with the linewidth of the generated wave, has been detected; that is, the linewidth of the wave remains negligible with respect to the stabilization uncertainty. However, as the frequency conversion becomes more efficient, the linewidth of the FWM grows accordingly. For higher conversion efficiencies, a trade-off would probably be established between the conversion efficiency and the uncertainty in the generated wave: Better conversion efficiency would come at the expense of a bigger linewidth in the new frequency. In our experiment we used lasers whose linewidths are not greater than $150 \mathrm{kHz}$, a value that is negligible in comparison with the stabilization uncertainties of the FWM pumps. Because the conversion efficiency is rather low, the linewidth of the FWM is still expected to be negligible compared with the uncertainty of the stabilization.

For such narrow-line-width lasers, another issue arises: Brillouin scattering in the optical fiber. This effect basically limits the amount of power that can be delivered into the fiber with our narrowlinewidth sources. For simplicity, this effect has been neglected in the theoretical treatment, although a more-precise model should include it. To overcome this problem and achieve more efficiency, phase dithering of the FWM pumps would be necessary, and thus the uncertainty that is due to the linewidth should grow drastically.

\section{Conclusions}

We have demonstrated that it is possible to generate new reference frequencies from other frequencies by means of fiber Raman-enhanced four-wave mixing. We have shown that our configuration is extremely

Table 1. Possible Reference Frequencies Compared with ITU Frequencies ${ }^{a}$

\begin{tabular}{|c|c|c|c|c|c|c|c|c|}
\hline \multicolumn{2}{|c|}{$f_{1}(\mathrm{GHz})^{b}$} & \multicolumn{2}{|c|}{$f_{2}(\mathrm{GHz})^{b}$} & \multirow{2}{*}{$\begin{array}{c}\begin{array}{c}f_{\mathrm{FWM}} \\
(\mathrm{GHz})\end{array} \\
191249.78\end{array}$} & \multirow{2}{*}{$\begin{array}{c}\begin{array}{c}f_{\mathrm{ITU}} \\
(\mathrm{GHz})\end{array} \\
191250\end{array}$} & \multirow{2}{*}{$\frac{\begin{array}{c}f_{\mathrm{FWM}}-f_{\mathrm{ITU}} \mid \\
(\mathrm{MHz})\end{array}}{220}$} & \multirow{2}{*}{$\frac{\begin{array}{r}\Delta f_{1} \\
(\mathrm{GHz})\end{array}}{0.1}$} & \multirow{2}{*}{$\frac{\begin{array}{c}\Delta f_{\mathrm{FWM}} \\
(\mathrm{GHz})\end{array}}{0.35}$} \\
\hline 197522.92 & (R18) & 194386.35 & $(P 27)$ & & & & & \\
\hline 197580.99 & (R15) & 194565.91 & $(P 25)$ & 191550.83 & 191550 & 830 & 0.1 & 0.35 \\
\hline 197282.75 & (R10) & 194565.91 & $(P 25)$ & 191849.07 & 191850 & 930 & 0.06 & 0.28 \\
\hline 196832.35 & $(R 3)$ & 194565.91 & $(P 25)$ & 192299.47 & 192300 & 530 & 0.06 & 0.28 \\
\hline 197404.40 & (R12) & 195001.93 & $(P 20)$ & 192599.46 & 192600 & 540 & 0.1 & 0.35 \\
\hline 196123.05 & $(P 6)$ & 194386.35 & $(P 27)$ & 192649.65 & 192650 & 350 & 0.09 & 0.33 \\
\hline 195971.98 & $(P 8)$ & 194386.35 & $(P 27)$ & 192800.72 & 192800 & 720 & 0.1 & 0.35 \\
\hline 195580.99 & $(P 13)$ & 194565.91 & $(P 25)$ & 193550.83 & 193550 & 830 & 0.05 & 0.26 \\
\hline
\end{tabular}

${ }^{a}$ In all of these cases, $\Delta f_{2}=0.1 \mathrm{GHz} ; f_{1}$ and $f_{2}$ are the FWM pump frequencies, $f_{\mathrm{FWM}}$ is the frequency of the four-wave mixing, and $\Delta f_{i}$ are the uncertainties of the corresponding frequencies.

${ }^{b}$ Numbers in parentheses are absorption lines. 
efficient for the generation of new references over a broad range of wavelengths. The uncertainty of the new frequency is basically comparable to the uncertainty of the stabilization of the original FWM pump waves.

We acknowledge financial support from the Spanish Ministerio de Ciencia y Tecnología through project TIC2000-2005. We are grateful to Jesús González of Telefónica de España for his interest in our study. M. González Herráez acknowledges support from the Comunidad Autónoma de Madrid through a Formación de Personal Investigador grant.

\section{References}

1. W. C. Swann and S. L. Gilbert, "Pressure-induced shift and broadening of 1510-1540 $\mathrm{nm}$ acetylene wavelength calibration lines," J. Opt. Soc. Am. B 17, 1263-1270 (2000).

2. K. Nakagawa, M. de Labachelerie, Y. Awaji, and M. Kourogi, "Accurate optical frequency atlas of the $1.5-\mu \mathrm{m}$ bands of acetylene,” J. Opt. Soc. Am. B 13, 2708-2714 (1996).
3. M. de Labachelerie, K. Nakagawa, and M. Ohtsu, "Ultranarrow ${ }^{13} \mathrm{C}_{2} \mathrm{H}_{2}$ saturated-absorption lines at $1.5 \mu \mathrm{m}$," Opt. Lett. 19, 840-842 (1994).

4. Y. Awaji, M. de Labachelerie, M. Ohtsu, and H. Sasada, "Optical frequency measurement of the $\mathrm{H}^{12} \mathrm{C}^{1} 4 N$ Lamb-dip-stabilized 1.5- $\mu$ m diode laser," Opt. Lett. 20, 2024-2026 (1995).

5. S. Trillo and S. Wabnitz, "Parametric and Raman amplification in birefringent fibers," J. Opt. Soc. Am. B 9, 1061-1082 (1992).

6. T. Sylvestre, H. Maillotte, E. Lantz, and T. Dinda, "Ramanassisted parametric frequency conversion in a normally dispersive single-mode fiber," Opt. Lett. 24, 1561-1563 (1999).

7. G. P. Agrawal, Nonlinear Fiber Optics (Academic, San Diego, Calif., 2001), Chap. 1.

8. K. J. Blow and D. Wood, "Theoretical description of transient stimulated Raman scattering in optical fibers," IEEE J. Quantum Electron. 25, 2665-2673 (1989).

9. S. L. Gilbert and W. C. Swam, "Standard Reference Material: Acetylene ${ }^{12} \mathrm{C}_{2} \mathrm{H}_{2}$ Absorption Refence for $1510 \mathrm{~nm}$ to $1540 \mathrm{~nm}$ Wavelength Calibration-SRM 2517a," NIST Spec. Publ. 260133 (National Institute of Standards and Technology, Gaithersburg, Md., 2001). 